\title{
Mejora participativa del hábitat en contextos de desigualdad en ciudades intermedias
}

\author{
Participatory habitat improvement in contexts of inequality in \\ medium-sized cities
}

\author{
Paula Boldrini ${ }^{1}$ y Matilde Malizia ${ }^{2}$ \\ Fecha de recepción: 12-03-2020 - Fecha de aceptación: 16-06-2020 \\ Hábitat y Sociedad (ISSN 2173-125X), n. ${ }^{\circ} 13$, noviembre de 2020, pp. 209-228. \\ http://dx.doi.org/10.12795/HabitatySociedad.2020.i13.12
}

\begin{abstract}
Summary
The conditions that are vulnerable urban varied qualitatively in the last ten years. To the already known prevailing inequality conditions are added the increase and consolidation of drug production, sale and consumption circuits, mainly paco, which generate and deepen various problems, structurally transforming regional, local and neighborhood socio-spatial dynamics. Given this situation, other modes of social organization arise, which bring with them new coping strategies, technical support (public, academic and non-governmental institutions, among others), design and implementation of public policies that seek to influence specific transformations in the territory. However, this scenario finds technicians mostly de-instrumented to intervene in the territory with the old strategies - already rudimentary and now also outdated - that require rigorous transformations to reach an appropriate social approach. That is why it is proposed as a main objective to identify those appropriate strategies for the integral improvement of the popular habitat through community participation and strengthening processes. For its development it is based on concrete experiences of technical support carried out in five vulnerable neighborhoods of the Gran San Miguel de Tucumán agglomerate, located in northwestern Argentina, since 2015.
\end{abstract}

\section{Key words}

Participatory strategies; Medium-sized cities; Vulnerable neighborhoods; Addictions; Gran San Miguel de Tucumán

\begin{abstract}
Resumen
Las condiciones en las que se encuentran los sectores vulnerables urbanos variaron cualitativamente en la última década. A las ya conocidas condiciones de desigualdad imperantes se suman el aumento y consolidación de circuitos de producción, venta y consumo de drogas, fundamentalmente de paco, que generan y profundizan diversas problemáticas transformando estructuralmente las dinámicas socioespaciales regionales, locales y barriales. Ante esta situación surgen otros modos de organización social, que traen aparejados nuevas estrategias de abordaje, acompañamiento técnico (instituciones públicas, académicas y ONG, entre otras), diseño y ejecución de políticas públicas que pretenden incidir en transformaciones concretas en el territorio. Sin embargo, este escenario encuentra a los técnicos mayormente des-instrumentados para intervenir en el territorio con las antiguas estrategias - ya rudimentarias y ahora también desactualizadas - que requieren rigurosas transformaciones para alcanzar un abordaje social apropiado. Así, se propone como objetivo principal identificar aquellas estrategias apropiadas para la mejora integral del hábitat popular mediante procesos de participación y fortalecimiento comunitario. Para su desarrollo se parte de experiencias concretas de acompañamiento técnico llevadas adelante en cinco barrios vulnerables del aglomerado Gran San Miguel de Tucumán, localizado en el noroeste argentino, desde el año 2015.
\end{abstract}

\section{Palabras clave}

Estrategias participativas; Ciudades intermedias; Barrios vulnerables; Adicciones; Gran San Miguel de Tucumán

1 Arquitecta, Doctora en Ciencias Sociales y Magister en Psicología Social, todos por la Universidad Nacional de Tucumán (UNT), Argentina. Investigadora Adjunta del Consejo Nacional de Investigaciones Científicas y Técnicas (CONICET). E-mail: paula_boldrini@hotmail.com. ORCID: 0000-0001-6311-9533.

2 Licenciada en Trabajo Social y Doctora en Ciencias Sociales con orientación en Geografía por la Universidad Nacional de Tucumán (UNT), Argentina. Investigadora Adjunta del Consejo Nacional de Investigaciones Científicas y Técnicas (CONICET). E-mail: matumalizia@yahoo.com.ar. ORCID: 0000-0002-0264-3451. 


\section{Introducción}

Las condiciones en las que se encuentran los sectores vulnerables urbanos, concentrados principalmente en asentamientos populares, han variado cualitativamente en los últimos diez años. Estos cambios conllevan el surgimiento de otros modos de organización social, que traen aparejados nuevas estrategias de abordaje, acompañamiento técnico (instituciones públicas, académicas y no gubernamentales, entre otras), diseño y ejecución de políticas públicas que pretenden incidir en transformaciones concretas en el territorio.

El aglomerado Gran San Miguel de Tucumán, considerado una ciudad intermedia en la jerarquía urbana nacional, integra la región denominada Norte Grande Argentino. ${ }^{3}$ Esta región se caracteriza por el elevado nivel de fragmentación de su estructura socio-espacial, concentra los índices de pobreza más elevados y los niveles más bajos de calidad de vida del país (Bolsi y Paolasso, 2009). ${ }^{4}$ A esta situación se suma en los últimos años el aumento y consolidación de circuitos de producción, venta y consumo de drogas, fundamentalmente de $p a c o,{ }^{5}$ que generan y profundizan diversas problemáticas transformando estructuralmente las dinámicas socio-espaciales regionales, locales y barriales. En particular, el avance de este fenómeno impacta en la cotidianeidad barrial a través de la consolidación de circuitos de narcomenudeo. Estos circuitos colonizan el espacio público y lo transforman en un ámbito inseguro, restringiendo las actividades al espacio estrictamente familiar, también vulnerado bajo condiciones de precariedad a la que se suma la hostilidad resultante de los efectos del consumo.

Estas problemáticas son identificadas como emergentes prioritarios por vecinos y técnicos que trabajan en el territorio, convirtiéndose en motor de procesos participativos. Sin embargo, este escenario encuentra a los técnicos mayormente des-instrumentados para intervenir en el territorio con las antiguas estrategias - ya rudimentarias y ahora también desactualizadas- que requieren rigurosas transformaciones para alcanzar un abordaje social apropiado.

Este artículo tiene como objetivo central identificar aquellas estrategias que resultan más apropiadas para trabajar en la mejora integral del hábitat popular mediante procesos de participación y fortalecimiento comunitario. En este sentido se busca que dichas estrategias se conviertan en un insumo teórico-instrumental para las lógicas y prácticas participativas implementadas en barrios en condiciones de vulnerabilidad. Para su desarrollo se consideraron cinco experiencias de acompañamiento técnico en barrios populares del aglomerado Gran San Miguel de Tucumán, desarrolladas por el Equipo Interdisciplinario Mejora del Hábitat Participativo (MHaPa) ${ }^{6}$ Las actividades se llevaron a cabo durante el periodo 2015-2019, aunque los vínculos, tareas y proyectos se mantienen hasta la actualidad. En ellas predominó la organización comunitaria participativa y el acompañamiento técnico institucional, tanto terapéutico como vinculado al desarrollo de acciones para transformar el hábitat y el espacio público de manera central.

El enfoque metodológico propuesto combinó el desarrollo de estrategias participativas con el uso de herramientas cualitativas. La investigación contempló los procesos barriales de manera comparativa, considerando su lugar en el contexto metropolitano, las problemáticas urbanas específicas y las condiciones socio-organizativas particulares. Los resultados obtenidos permitieron identificar similitudes y diferen- 
cias entre los casos estudiados, y vinculaciones entre estos procesos y los fenómenos urbanos asociados a la desigualdad.

\section{Procesos de mejora barrial en contextos de pobreza}

Los procesos de mejora barrial que tienden a perdurar y resolver con mayor efectividad los problemas del hábitat son aquellos que se apoyan en procesos participativos que garantizan la pertinencia y apropiación de las medidas de mejora implementadas (Pelli, 2007; Boldrini, 2012; Enet, 2012). No obstante, existe una contracara de la participación, es aquella que se sustenta en el disciplinamiento basado en una interacción compleja entre diversas formas de control, con un dominio espacial de tipo intensivo. Esta última es la que ha dominado la ejecución de las políticas públicas de vivienda en las últimas décadas en Argentina y Latinoamérica (Duhau, 1998; Boldrini, 2012). Se deben distinguir los tipos de control llevados adelante mediante dinámicos procesos de des-reterritorialización (Haesbaert, 2011) de las formas de resistencia a las lógicas de la ciudad neoliberal (Janoschka, 2011). En este último aspecto se inscriben los estudios llevados a cabo por autores como Castells (1985 y 1968), Pradilla (1984), Duhau (1998), Auyero (2002) y Harvey (2013) que centran su interés en los movimientos sociales de acceso al suelo urbano y en la redefinición de nuevas formas de control social y político de las demandas populares, pasando progresivamente de la lucha reivindicativa a la propositiva (Coulomb, 1992) y cuestionando la legitimidad de la gestión urbana existente (Cravino, 2008). Es por ello que resulta imprescindible la realización de esfuerzos adicionales que pongan en discusión nuevas alternativas para re-apropiar el medio urbano mediante el recurso de la participación como una práctica dominante de ciudadanía urbana (Purcell, 2007; Ciccolella, 2010; Pradilla, 2010; Boldrini, 2011).

Durante la última década las condiciones de vida en los barrios populares estuvieron - y continúan estando-condicionadas por el impacto del consumo, venta y producción de drogas, que alteran y reestructuran los usos, percepciones y lógicas cotidianas (Boldrini, 2018). La problemática inherente a las drogas es un fenómeno multicausal que ocupa un lugar prioritario en estos barrios. La manera de enfrentar esta problemática (intercambio y consumo de drogas) se aborda desde dos paradigmas opuestos: el prohibicionista y el de reducción de daños (Llovera y Scialla, 2017). El primero está orientado a la culpabilización y persecución tanto de vendedores como de consumidores de drogas, seguido de un tratamiento manicomial del sujeto adicto. Por el contrario, la lógica de reducción de daños apunta a separar la venta del consumo, atendiendo a este último desde una perspectiva multicausal y en el ámbito donde se desarrolla la cotidianeidad de los sujetos adictos. Esto conlleva un cambio radical en las políticas públicas de prevención y tratamiento de adicciones junto a un re-encuadre estructural tanto de las medidas concretas como de la formación específica de los técnicos en territorio.

Las estrategias participativas deben adaptarse al contexto actual y su efectividad depende de la construcción de vínculos y la primacía de la integración comunitaria (Boldrini, 2012). La participación conlleva el desarrollo de actividades en ámbitos apropiados, alcanzando — bajo condiciones de escasa gobernabilidad- instancias de protesta que evidencian rasgos repolitizantes de estas prácticas; en una dinámica ca- 
paz de atravesar una espiral dialéctica donde cada experiencia retroalimenta un proceso de maduración y fortalecimiento progresivo a través del tiempo. En la actualidad, para emprender transformaciones en los barrios populares atravesados por la problemática de las drogas como principal - pero no único- emergente es preciso conocer y entender esta situación. Esto permitirá proponer y realizar acciones desde la lógica participativa tendientes a la mejora cualitativa integral del hábitat, cuyos resultados impactarán tanto en el espacio concreto de estos barrios como en la calidad de vida de sus habitantes.

\section{La ciudad en la que se desarrollan los casos de estudio}

Los territorios que integran el Norte Grande Argentino son muy diferentes unos de otros y han sido ocupados de manera diferencial por distintas sociedades a través del tiempo. Esta región comparte una estructura socio-espacial fragmentada que se manifiesta en desigualdades que van desde el desequilibrio en la ocupación de su territorio - con el despoblamiento rural forzado-, hasta la configuración de ciudades excluyentes que reproducen las lógicas urbanas latinoamericanas.

El proceso de conformación del aglomerado Gran San Miguel de Tucumán se caracterizó por un desarrollo urbano condicionado por la actividad azucarera en la provincia y su posterior crisis en la década de los sesenta (Osatinsky y Paolasso, 2012). En la actualidad su proceso de crecimiento y expansión se encuentra determinado, principalmente, por el accionar de distintos actores (sobre todo privados) que conducen la ocupación del territorio en función de sus características ambientales (Paolasso, Del Castillo, Malizia y Boldrini, 2013), dando como resultado una expansión en forma de islas inconexas (Paolasso, Malizia y Longhi, 2011; Gómez López, Cuozzo y Boldrini, 2012). Dado su número de habitantes (890804 personas según la Encuesta Permanente de Hogares, cuarto trimestre de 2018), es la ciudad intermedia más poblada de la región, destacándose como nodo central.

La propagación de los barrios populares (villas, asentamientos informales y conjuntos de vivienda pública precarizados) ${ }^{7}$ en el aglomerado contribuyó a su conformación actual. Este tuvo como primer motor la migración campo-ciudad derivada del cierre masivo de los ingenios azucareros que desmanteló la estructura productiva de la provincia durante las décadas de los sesenta y setenta. El segundo momento se identifica a fines de la década de los ochenta como resultado del predominio de la aplicación de políticas neoliberales que provocaron un significativo aumento espacial y poblacional de los asentamientos informales vinculados, sobre todo, a la migración intraurbana de grupos sociales empobrecidos. Finalmente, a partir del año 2003 se detectó un tercer momento que se caracteriza por la desaceleración del crecimiento sostenido de los asentamientos informales a expensas del aumento de barrios promovidos desde el Estado dirigidos a sectores populares (Boldrini, 2018). Sin embargo, los grupos relocalizados mantienen problemáticas de profunda vulnerabilidad en su nuevo espacio (Gómez López, Cuozzo y Boldrini, 2015). Se estima que durante el periodo 1970-2014 su superficie urbanizada se duplicó mientras que los asentamientos informales crecieron un 400\% (Boldrini, 2011; Gómez López, Cuozzo y Boldrini, 2012).

Los barrios populares seleccionados son un claro ejemplo de este derrotero. Se localizan en las zonas más degradas del aglomerado (Fi- 
gura 1). Si bien el entorno en que se sitúan estos barrios se caracteriza, principalmente, por la informalidad y la pobreza (Boldrini, 2018), los dos primeros forman parte de un arco de pobreza situado hacia el este del aglomerado, que concentra la población con peores condiciones de vida y, por lo tanto, donde predominan los asentamientos informales y villas miseria (Boldrini, Del Castillo y Malizia, 2014). Dada su localización, estos barrios se encuentran desconectados en términos socioespaciales de la ciudad formal; no poseen espacios públicos o bien no están calificados o cualificados debidamente; tienen equipamiento comunitario escaso y deficitario; y, por lo general, su acceso a los servicios básicos es limitado en tanto la provisión de agua potable es clandestina, no poseen cloacas y no tienen recolección de residuos, situaciones que ponen en riesgo la salud de quienes viven allí. Además, sus habitantes tienen importantes problemas laborales, educativos y habitacionales. Como explica Del Castillo (2012), vivir en estos barrios constituye una estrategia intergeneracional para dar respuesta a sus necesidades de reproducción social.

La localización sectorizada de los diferentes tipos de barrios que se presenta en la Figura 1 pone en evidencia la división social del espacio
Figura 1. Localización de los barrios Costanera, Los Vásquez, Santa Inés, 11 de Enero y Diagonal Norte en el aglomerado Gran San Miguel de Tucumán (Norte Grande Argentino). Fuente: Bases de datos de Malizia y Boldrini (2017) y del Programa de Mejora del Hábitat Participativo.

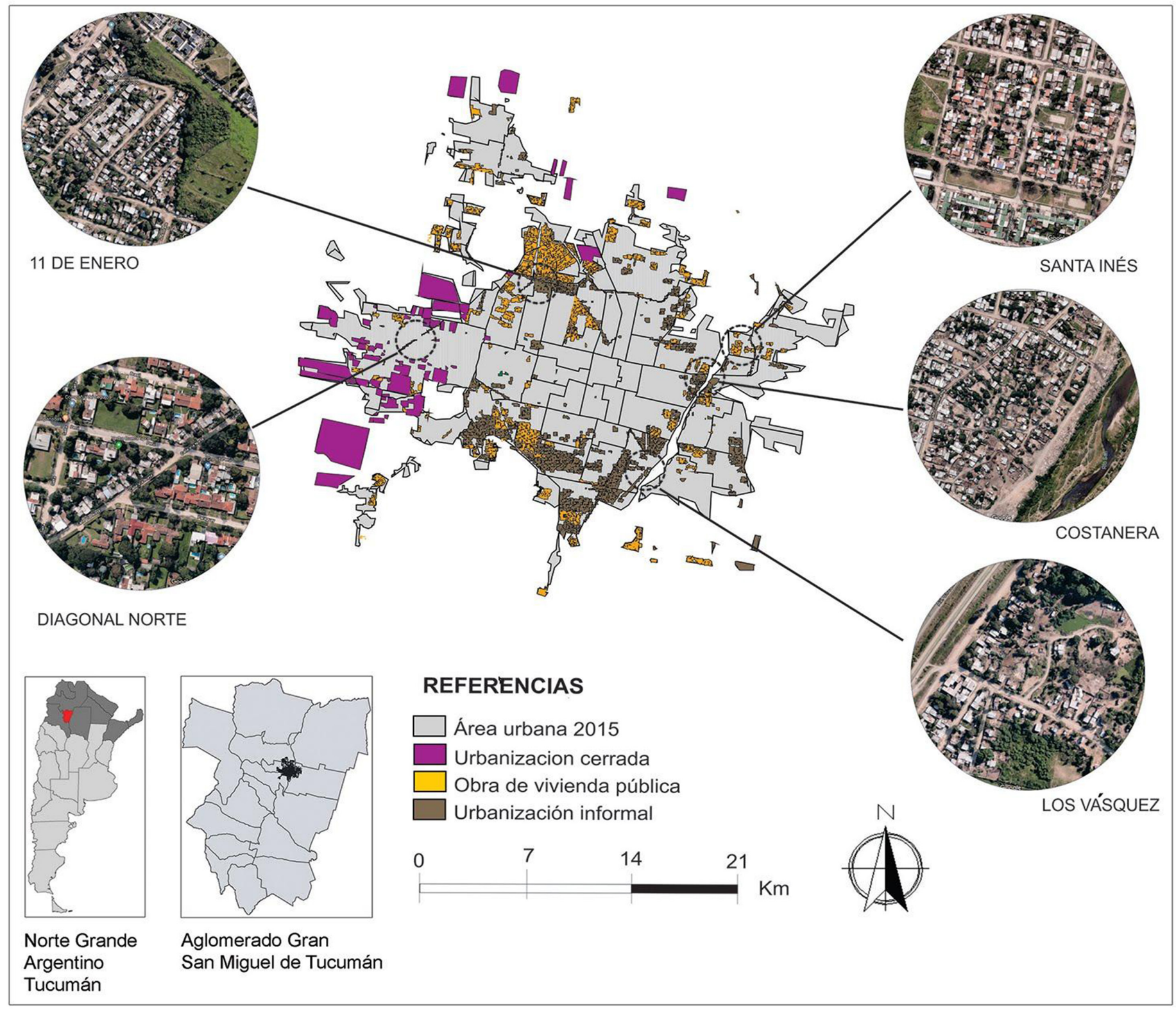


del aglomerado vinculada fuertemente con la dimensión socio-económica -ingresos e inserción en el mercado laboral formal e informal de sus habitantes- La desigual distribución y apropiación de las distintas partes que conforman el aglomerado se relaciona con aspectos tales como la provisión de infraestructuras, accesibilidad, calidad y tipo de viviendas, espacios de esparcimiento, equipamientos comunitarios y estatus simbólico que implica vivir en determinados barrios (por ejemplo, en las urbanizaciones cerradas) y contribuye a la configuración de una ciudad profundamente fragmentada. La obra de vivienda pública y la urbanización informal (villas y asentamientos informales) se distribuyen de manera similar conformando un arco norte-este-sur, y ambas están destinadas a grupos con recursos económicos bajo, medio-bajo y medio. En cambio, en la zona oeste del aglomerado, caracterizada por una mejor calidad urbano-ambiental, prevalecen las urbanizaciones cerradas destinadas a sectores con mayor poder adquisitivo.

\section{Consideraciones metodológicas}

El enfoque metodológico propuesto combina el desarrollo de estrategias participativas (Boldrini, 2012) con el uso de herramientas cualicuantitativas (Vasilachis de Gialdino, 2006; Mendizábal, 2019). Utilizar ambas metodologías en forma conjunta para trabajar con grupos vulnerables implica la posibilidad de unir el mundo académico con la práctica concreta (Svampa, 2008) mediante el continuo entrelazamiento entre el marco teórico-conceptual y la realidad estudiada (Althabe, 1999).

La implementación de estrategias participativas es una modalidad mediante la cual se emprende un proceso de mejora del hábitat - tangible e intangible - de un grupo o comunidad a partir de su propia iniciativa, la cual mantiene un ejercicio activo y sostenido en el tiempo, mediante ámbitos y prácticas apropiadas y transformadoras, a través de las cuales se construyen vínculos interpersonales capaces de mantener el predominio de la integración comunitaria. Incluye tres variables fundamentales: la construcción de vínculos, los ámbitos donde se desarrolla el proceso y la temporalidad (Boldrini, 2012).

La aplicación de herramientas cuali-cuantitativas permite el estudio, uso y recolección de una variedad de fuentes de información que describen los momentos habituales, problemáticos y significativos de la vida de los sujetos (Vasilachis de Gialdino, 2006). Entre las mismas se destaca el uso de observación con participación (Guber, 2009), entrevistas y encuestas sociales (Neiman y Quaranta, 2006) y análisis de documentos bibliográficos, catastrales, oficiales y periodísticos (Yuni y Urbano, 2003). ${ }^{8}$

Para su desarrollo se seleccionaron cinco barrios populares con condiciones de vulnerabilidad localizados en forma dispersa en el aglomerado Gran San Miguel de Tucumán: Los Vásquez, Diagonal Norte, Santa Inés, 11 de Enero y Costanera. En el primer barrio el trabajo de campo comenzó en el año 2015, en el segundo en el año 2016 y en los últimos tres en 2017. Las actividades y tareas se mantienen hasta la actualidad en el marco del Equipo MHaPa (salvo en Costanera donde la situación de violencia permanente dificultó el acceso a partir de comienzos de 2019 y en Santa Inés donde las actividades se suspendieron temporalmente desde mediados de 2019). Durante el mismo se realizaron múltiples entrevistas y encuestas sociales (53 en Los Vásquez, 56 en Diagonal Norte, 21 en Santa Inés y 35 en 11 de Enero), 25 talleres

8 Por razones de confidencialidad no se revelan los nombres de los encuestados, solo se los menciona por su inicial. 
(con un promedio de 5 talleres por barrios) en los que participaron en forma intermitente los habitantes de los barrios, diversas reuniones grupales y asambleas, acompañadas en todo momento por observación con participación para obtener notas de campo. ${ }^{9}$ Asimismo, en estos barrios se realizan diversas festividades como el día del niño, bingos y loterías solidarias y mingas de trabajo en lo que también se participó para fortalecer y consolidar los vínculos con sus residentes.

Durante la ejecución de las distintas actividades la mirada se centró tanto en aspectos generales como particulares de cada barrio teniendo en cuenta caracterización y descripción de las condiciones poblacionales; presencia de instituciones estatales y organizaciones sociales; identificación de las principales problemáticas vinculadas con la presencia de drogas; y análisis del proceso de mejora participativa a partir de tres aspectos claves: temporalidad, construcción de vínculos y ámbitos de trabajo.

\section{Presentación de los resultados}

Los resultados obtenidos fueron organizados en cinco apartados. El primero incluye la caracterización de los barrios seleccionados teniendo en cuenta sus particularidades. En el siguiente se realiza una descripción y análisis de las condiciones poblacionales y de la presencia de organizaciones sociales e instituciones estatales. En el tercer apartado, se identifican las problemáticas vinculadas con la droga, afectadas significativamente por la incorporación de las dinámicas de consumo y ventas. Luego se analizan los procesos de mejora participativa del hábitat desarrollados en cada barrio teniendo en cuenta, sobre todo, las variables temporalidad, construcción de vínculos y ámbitos de trabajo. Y por último se presenta una síntesis de los resultados que conduce al planteamiento de lineamientos estratégicos vinculados con la problemática tratada.

\section{Presentación y caracterización de los barrios populares estudiados}

Los barrios Costanera, Los Vásquez y Santa Inés se localizan hacia el este del aglomerado, en un sector caracterizado según Cuozzo, Boldrini y Malizia (2018) por la presencia de grandes islas residenciales de carácter homogéneo, ${ }^{10}$ en un área donde predominan los barrios populares. Por el contrario, los barrios 11 de Enero y Diagonal Norte su ubican hacia el oeste, en un área identificada como residencial atomizada de carácter mixto, en tanto allí conviven sectores sociales con diferente poder adquisitivo.

En el barrio Costanera el proceso de ocupación comenzó a fines de la década de los sesenta, y hoy abarca más de 50 manzanas irregulares, sectorizadas por áreas entre las que se encuentra Costanera Norte, sector en el que se realiza esta investigación. Su origen se enmarca en el proceso de desmantelamiento de la estructura productiva azucarera, que provocó movimientos migratorios rural-urbanos de trabajadores desocupados, ocasionando un proceso de asentamiento gradual por autoproducción del hábitat. Las viviendas se encuentran distribuidas en forma irregular con un patrón de asentamiento laberíntico, donde la conexión entre dos puntos se resuelve a través de pasadizos o callejones (Neder, Caria y Busnelli, 2008).
9 En algunas de estas actividades participaron los alumnos de la materia Práctica Profesional Asistida "Hábitat Participativo" de la Facultad de Arquitectura y Urbanismo (Universidad Nacional de Tucumán) dictada en el marco del Equipo MHaPa.

10 Cuozzo, Boldrini y Malizia (2018) definen tres tipos de áreas urbanas: grandes islas residenciales; residencial atomizado o pequeñas islas residenciales; y tradicional contemporáneo. 
El barrio Los Vásquez tiene una dinámica similar. Se inició mediante la ocupación informal de tierras en el año 1986 y abarca 5 manzanas irregulares que se distribuyen en torno al ex vaciadero de residuos sólidos urbanos, cerrado en el año 2005, aunque aún funcionan los hornos destinados a la quema de residuos patológicos. Cuenta con un merendero barrial a cargo de un grupo de jóvenes en proceso de recuperación de las adicciones.

En ambos barrios se llevan a cabo merenderos y comederos sociales como parte de las actividades y estrategias de reinserción social implementadas por los equipos técnicos de los dispositivos de salud pertenecientes a la Secretaría de Estado de Prevención y Asistencia de las Adicciones del Ministerio de Desarrollo Social Provincial. Además estos barrios comparten un aspecto estructurante que se vincula con su ubicación sobre la ribera del río Salí. ${ }^{11}$ Son linderos a la ciudad consolidada, aun cuando el primero forma parte de las villas miseria originadas en la década de los sesenta y el segundo es un asentamiento informal surgido a mediados de la década de los ochenta. Su localización, en la zona más degrada y contaminada del aglomerado, contribuye a que sus condiciones de pobreza se encuentren entre las más vulnerables del Gran San Miguel de Tucumán.

El barrio Santa Inés se inició en el año 1998 mediante la entrega de casas por parte del Instituto Provincial de la Vivienda y Desarrollo Urbano y abarca 8 manzanas irregulares. Sin embargo, estas viviendas fueron entregadas a las familias antes de que finalizara su construcción con diversos problemas estructurales y de conexión a servicios básicos, que se mantienen hasta la actualidad. De este modo, presenta condiciones de vulnerabilidad que lo equiparan con cualquier asentamiento informal regularizado. El barrio cuenta con la presencia de la Asociación Civil Solidaria de Alderetes que coordina, desde hace veinte años, diversas actividades, en articulación con los Técnicos de los dispositivos de salud en adicciones.

El barrio 11 de Enero se inició mediante la ocupación informal de tierras en el año 2004 y abarca 13 manzanas irregulares hoy consolidadas. Posteriormente en el año 2007 hubo un intento de relocalización de estas familias en otros barrios próximos, no obstante, los terrenos desocupados fueron re-ocupados. Cuenta con un merendero y una cooperativa de trabajo que elabora alimentos para la venta, cuyas ganancias sostienen el merendero, que carece de financiamiento estatal.

El barrio Diagonal Norte se originó en el transcurso de la década de los sesenta en 12 manzanas longitudinales sobre las ex vías de ferrocarril. Se localiza en el municipio Yerba Buena que se caracteriza por su elevada calidad ambiental y por la concentración de urbanizaciones cerradas (Malizia y Boldrini, 2012; Malizia, 2011). Si bien esto podría leerse como un contexto ventajoso, resulta contradictorio, en tanto se encuentra desarticulado de la trama urbana consolidada, encerrados por las medianeras de las urbanizaciones cerradas que evaden su existencia. En el barrio funciona el club de veteranos El Sapito, desde la década de los setenta, que incluye a todas las categorías de la liga de fútbol.

En estos dos barrios también se llevan a cabo diversas actividades entre las que se encuentran merenderos y comedores que son organizados y sostenidos en forma conjunta por la comunidad y la Organización Social La Poderosa.

En cuanto a las características laborales, los habitantes de Costanera y Los Vásquez viven del trabajo precarizado e informal. En Costane- 
ra prevalecen las actividades de cartoneo, debido a su cercanía al principal al centro de la ciudad, y en los Vásquez predominan las tareas asociadas a la separación de residuos resultado de su localización colindante al ex vaciadero de residuos sólidos urbanos y a áreas vecinas en las que se continúan arrojando residuos de modo informal. En los barrios 11 de Enero, Santa Inés y Diagonal Norte predomina el trabajo informal en viviendas particulares de la zona (limpieza, jardinería y cuidado de personas) y en menor medida obreros de la construcción y pequeños comercios barriales.

\section{Condiciones poblacionales y presencia de organizaciones e instituciones}

Este apartado se basa en el análisis de la relación entre las condiciones de la población — teniendo en cuenta las variables sujeto, grupo y comunidad (Pichon-Rivière, 1999) - y la presencia de las organizaciones sociales e instituciones estatales que motorizan el proceso participativo (Cuadro 1). En Costanera, Los Vásquez y Santa Inés, en los que prevalecen condiciones de deterioro social extremo, están trabajando en forma activa los técnicos de los dispositivos de salud que dinamizan las experiencias grupales a partir de estrategias terapéuticas. En cambio, en los barrios 11 de Enero y Diagonal Norte, los referentes de la organización barrial La Poderosa promueven diversas actividades colectivas en un contexto en el que la problemática de las adicciones es menor, las condiciones sociales son menos graves y los conflictos intrabarriales e interpersonales son de menor envergadura. Si bien en todos los casos el interés colectivo se orienta a la mejora del espacio público, en los tres primeros casos la demanda busca revertir un uso del espacio público tomado por transas ${ }^{12}$ y grupos de jóvenes en consumo, mientras que en los dos últimos la demanda tiene un carácter más preventivo.

En todos los casos, el Equipo MHaPa interviene en actividades vinculadas con la mejora del hábitat y el desarrollo de procesos de vinculación tecnológica que redundan en el aporte instrumental de los procesos estudiados. Este grupo desarrolla y acompaña, mediante lógicas participativas, procesos proyectuales de espacio público, relevamientos socio-espaciales y armado de carpetas técnicas elevadas a instituciones públicas para su tratamiento o, como el caso de Diagonal Norte, la contribución de datos centrales y parte de la redacción del proyecto de Declaración de Utilidad Pública sujeto a Expropiación del predio donde funciona el club El Sapito.

En el Cuadro 1 se puede observar los modos en que actuaron tanto los técnicos de las organizaciones sociales como de las instituciones estatales a través del apoyo a sus iniciativas en los barrios. Sin embargo, en esta instancia se separa el rol de los técnicos integrantes de los dispositivos de salud, de su propia institución pública. Estos técnicos operan bajo el paradigma de reducción de daños, aun cuando la mayoría de las instituciones públicas se rige bajo lógicas prohibicionistas, lo que impacta de manera conflictiva en las relaciones de trabajo entre los técnicos y la institución.

El Estado, tanto provincial como nacional, intervino mediante diferentes estrategias y una restringida gama de recursos, sobre todo, en los barrios Costanera y Los Vásquez. A pesar de estas restricciones, se lograron avances visibles de la mano de los dispositivos de salud en adicciones, con la conformación de grupos, proyectos colaborativos y
12 Término mediante el cual se conoce a las personas que venden drogas en los barrios populares. 
13 A fines del año 2016 cesaron los contratos de los profesionales que integraban los dispositivos de salud. Se apartó a los trabajadores que habían construido vínculos y procesos grupales probadamente exitosos, y se los sustituyó por profesionales sin experiencia que desconocían las tareas desarrolladas hasta el momento.

14 En el año 2014 el gobierno nacional anunció, como parte del programa "recuperar inclusión" (http://www.cfkargentina.com/cfk-en-twitter-lanzamientorecuperar-inclusion/), la construcción de un CEPLA en barrio Costanera, obra que comenzó en el año 2015 y se paralizó en el mismo año, con un avance del $43 \%$. No obstante, a principios del año 2018, el gobierno provincial confirmó el reanudamiento de las obras para concluir la construcción del CEPLA (http://www. tucumanalas7.com.ar/local/2018/3/15/ juan-manzur-anuncio-provincia-construira-cepla-171360.html). Desde el año 2019 el edificio está construido y funcionando, con quejas por parte de los vecinos sobre su eficacia en relación al problema específico de la droga para el que fue creado. Toda la gestión se encuentra dentro de la esfera de las Secretarías de Prevención y Asistencia de las Adicciones (provincial) y de Programación para la Prevención de la Drogadicción y la Lucha contra el Narcotráfico (nacional).

15 Por su parte el Club Social y Deportivo de Los Vásquez fue seleccionado en el año 2016 por las Secretarías de Juventud Nacional y Provincial para ser construido, sujeto a múltiples disputas entre estas esferas para ser luego abandonado y desconocido como proyecto colectivo.

16 La organización del barrio, en torno a los dispositivos de adicciones, había proyectado la mejora de la plaza junto a los técnicos CONICET-UNT. La carpeta técnica había sido oportunamente presentada por mesa de entradas de la Municipalidad, y se encontraba perdida al momento de la irrupción sin previo aviso de la cooperativa de construcción que intentó iniciar obras en la plaza por orden del intendente articulaciones inter-barriales que evidenciaban su carácter repolitizante. No obstante, los resultados obtenidos nada tienen que ver con el aprovechamiento de los procesos de participación desarrollados. Por el contrario, allí donde surgía algún tipo de organización grupal y/o barrial con la suficiente fuerza para emprender cambios y/o mejoras en sus comunidades, el Estado, mediante diversas estrategias, los fue boicoteando y desmantelando sistemáticamente, aun cuando se tratara de los técnicos de su propia gestión. ${ }^{13}$ Esto se manifestó también cuando algunas instituciones públicas tanto de orden provincial como nacional se comprometieron a financiar dos proyectos vinculados a la problemática de adicciones que incluían la mejora del hábitat, el Centro Preventivo Local de Adicciones (CEPLA) ${ }^{14}$ en Costanera y el Club Social y Deportivo en Los Vásquez ${ }^{15}$ con un importante componente participativo por parte de la comunidad; sin embargo mientras el primero se ejecutó de espaldas a las demandas de los vecinos y de los propios dispositivos de salud, el segundo no tuvo apoyo económico y quedó en manos exclusivas de la autoconstrucción barrial, situación que provocó retrocesos en los procesos participativos desarrollados. Al respecto jóvenes de los distintos barrios expresaron: “[...] habían abandonado el CEPLA a medio construir, lo habían dejado robar entero, quedaba nada, pero cuando vieron que nos estábamos apropiando del espacio, se apuraron a ocuparlo y hoy no formamos parte de eso" (R., barrio Costanera, 2018); "Veníamos trabajando todos los días, cuando nos prometieron ayuda pudimos relajarnos, pero lo único que pasó es que todo quedó parado" (J., barrio Los Vásquez, 2017).

En el caso del barrio Santa Inés una organización con mucha antigüedad, la Asociación Civil Solidaria de Alderetes, sirve de apoyo a las actividades planteadas desde el dispositivo de salud, contribuyendo de manera dinámica a la conexión entre el grupo de jóvenes en proceso de recuperación y las familias del barrio. Allí también la mejora de una plaza sirve de aglutinante para la tarea colectiva, fundamentalmente autogestiva hasta el momento en que interviene el Municipio. Su intervención se produce interrumpiendo el proceso mediante un intento de apropiación de la mejora del espacio, que fue detenido por los vecinos que solicitan a las autoridades se atengan al proyecto comunitario. Una vecina comentaba: "[...] entonces el intendente, cuando vio que avanzábamos nosotros solos con la plaza vino a poner su cartel y meter cosas de prepo, pero no lo dejamos entrar y le dijimos, o respeta el proyecto, o a la plaza no entra" (A. L., barrio Santa Inés, 2018). ${ }^{16}$ En esta oportunidad se acepta lo propuesto por los habitantes del barrio y se produce un reacomodamiento en la tarea, las acciones desarrolladas por el municipio respetan el proyecto barrial, y disponen de los recursos para ejecutar la obra en coordinación con los técnicos del Equipo MHaPa.

En el caso de Diagonal Norte sucedió un comportamiento particular, el Estado a través del Municipio reúne las demandas barriales presentadas a través de proyectos técnicos, y utiliza una estrategia de sobrecarga de la comunidad para dilatar las respuestas y soluciones. Esto se expresa en una transferencia permanente de responsabilidades hacia los vecinos: “... ustedes también como organización no se mueven, si el estadito no les da algo..." (F. M., Secretaría de Obras Públicas y Planeamiento Urbano, Municipalidad de Yerba Buena). De esta forma el Estado se corre de su rol, aludiendo diferentes argumentos. Un vecino comentaba: "Vamos a las reuniones y nos quieren hacer creer que son indefensos, pero nosotros sabemos que el problema es que no somos 
una prioridad" (M., barrio Diagonal Norte, 2018). Pese a esto, los habitantes del barrio Diagonal Norte continúan las acciones entabladas por lograr que la Declaración de Utilidad Pública Sujeta a Expropiación del predio donde funciona el club deportivo El Sapito se concrete.

En el caso del barrio 11 de Enero las instituciones públicas se desligan de responsabilidad en tanto el espacio de la plaza, y parte del barrio se presenta como un área de pertenencia administrativa confusa, fuera de la jurisdicción de los dos municipios en cuyos límites se aloja. Esta condición desalienta las posibilidades de recepción de apoyo público, lo que impacta negativamente en el avance de la organización vecinal.

Si bien los barrios Santa Inés y Diagonal Norte cuentan con organizaciones sociales maduras que respaldan y promueven las actividades emprendidas, tienen condiciones excepcionales para la evolución de estas prácticas mediante el desarrollo de experiencias cada vez más consolidadas. No obstante, en todos los casos el Estado aparece como obstaculizador de las iniciativas barriales, aun cuando los técnicos mantienen una lógica contrapuesta.

\begin{tabular}{|c|c|c|c|c|c|}
\hline \multirow{2}{*}{ VARIABLES } & \multicolumn{5}{|c|}{ BARRIOS POPULARES } \\
\hline & Costanera & Los Vásquez & Santa Inés & 11 de Enero & Diagonal Norte \\
\hline \multirow{2}{*}{$\begin{array}{l}\text { Sujeto, grupo } \\
\text { y comunidad }\end{array}$} & \multicolumn{2}{|c|}{$\begin{array}{l}\text { Jóvenes afectados por el consumo de } \\
\text { drogas masivo, con elevado deterioro } \\
\text { físico y mental. Comunidad dividida y } \\
\text { enfrentada. Presencia de punteros po- } \\
\text { líticos }\end{array}$} & $\begin{array}{l}\text { Jóvenes con adic- } \\
\text { ciones. Muje- } \\
\text { res organizadas. } \\
\text { Comunidad con } \\
\text { buen nivel de in- } \\
\text { tegración }\end{array}$ & $\begin{array}{l}\text { Jóvenes con adic- } \\
\text { ciones. Falta de } \\
\text { comunicación y } \\
\text { cohesión barrial }\end{array}$ & $\begin{array}{l}\text { Jóvenes con adic- } \\
\text { ciones. Comuni- } \\
\text { dad con elevado } \\
\text { nivel de integra- } \\
\text { ción, internas ba- } \\
\text { rriales }\end{array}$ \\
\hline & \multicolumn{2}{|c|}{$\begin{array}{l}\text { Variable asistencia a los encuentros, es- } \\
\text { casas condiciones para el sostenimien- } \\
\text { to y ejecución de tareas conjuntas }\end{array}$} & $\begin{array}{l}\text { Variable asisten- } \\
\text { cia a los encuen- } \\
\text { tros, numerosas } \\
\text { condiciones para } \\
\text { el sostenimiento } \\
\text { y ejecución de ta- } \\
\text { reas conjuntas }\end{array}$ & $\begin{array}{l}\text { Escasa asistencia } \\
\text { a los encuentros, } \\
\text { malas condicio- } \\
\text { nes para el sos- } \\
\text { tenimiento y eje- } \\
\text { cución de tareas } \\
\text { conjuntas }\end{array}$ & $\begin{array}{l}\text { Elevada asistencia } \\
\text { a los encuentros, } \\
\text { numerosas condi- } \\
\text { ciones para el sos- } \\
\text { tenimiento y eje- } \\
\text { cución de tareas } \\
\text { conjuntas }\end{array}$ \\
\hline \multirow{4}{*}{$\begin{array}{l}\text { Organizacio- } \\
\text { nes / Insti- } \\
\text { tuciones que } \\
\text { participan }\end{array}$} & \multicolumn{3}{|c|}{ Dispositivos de Salud } & \multicolumn{2}{|c|}{ Organización Social La Poderosa } \\
\hline & $\begin{array}{l}\text { Grupo de jóvenes } \\
\text { "Ganas de Vivir" }\end{array}$ & $\begin{array}{l}\text { Grupo de jóvenes } \\
\text { "Con esperanza } \\
\text { nos fortalecemos" }\end{array}$ & $\begin{array}{l}\text { Asociación Civil } \\
\text { Solidaria de Alde- } \\
\text { retes }\end{array}$ & & $\begin{array}{l}\text { Club de Vetera- } \\
\text { nos "El Sapito" }\end{array}$ \\
\hline & $\begin{array}{l}\text { Mesa de Gestión } \\
\text { provincial (múlti- } \\
\text { ples programas y } \\
\text { equipos técnicos } \\
\text { del Estado, inclui- } \\
\text { do PROMEBA) }\end{array}$ & $\begin{array}{l}\text { Mesa de Gestión } \\
\text { provincial (refe- } \\
\text { rentes técnicos de } \\
\text { diferentes Progra- } \\
\text { mas del Estado) }\end{array}$ & \multicolumn{3}{|c|}{ Escasa presencia estatal a través de los municipios } \\
\hline & \multicolumn{5}{|c|}{ Equipo Interdisciplinario MHaPa. } \\
\hline
\end{tabular}

\section{Problemáticas vinculadas con la droga}

El período de austeridad y crisis económica por el que está transitando Argentina tiene repercusiones entre las que prevalecen aquellas referidas al avance del consumo, venta y producción de drogas en la sociedad. Los medios de comunicación tanto hegemónicos como alternativos — bajo lecturas contrapuestas- ponen a esta problemática en-
Cuadro 1. Condiciones poblacionales y presencia de organizaciones e instituciones. Fuente: Base de datos del Programa de Mejora del Hábitat Participativo 
tre sus temáticas más recurrentes (Décima, 2019). El coordinador de los Dispositivos de Salud explicaba:

[...] inicialmente el consumo de drogas en Tucumán era a través de inhalantes y pegamentos, siendo hasta aquel momento la Argentina una ruta de paso desde Perú y Bolivia. Luego de la crisis del 2001, aumentó el desarrollo y fortalecimiento del narcotráfico, con el que aparece el consumo de pasta base en los barrios vulnerables caracterizada por un uso adolescente, acrecentando la necesidad de asistencia de adicciones (E. M., Coordinador de los Dispositivos de Salud, 2017).

De acuerdo con el entrevistado, en el año 2006 aparecieron las primeras cocinas de cocaína y la venta de este producto era llevada a cabo por sujetos externos a los barrios. Esta situación provocó que los familiares de las personas en consumo pusieran en marcha diversas dinámicas para combatir a los vendedores, dinámicas que redundaron en el fortalecimiento de vínculos entre la comunidad en general y las madres en particular. En el año 2009 la profundización de esta problemática provocó el surgimiento de la organización Madres de Pañuelo Negro, integrada por madres que perdieron a sus hijos por suicidios o asesinatos de transas por deudas. Fundada para combatir la venta de drogas y denunciar a los transas, articula acciones con otras organizaciones como el Movimiento Madres de la Esperanza y la Hermandad de los Barrios que denuncian sistemáticamente la falta de políticas públicas para el abordaje de las adiciones.

A partir del año 2010, la forma de comercializar estas drogas en los barrios populares se transformó dando origen al narcomenudeo, es decir, a la venta de drogas en pequeñas cantidades ya no por agentes externos sino por los propios vecinos de los barrios. Esta modalidad incorpora nuevas estrategias que involucran a familias enteras en la comercialización de las drogas, y provoca un fuerte retroceso social que afecta la estructura social desde el seno familiar, hasta llegar a la descomposición de vínculos y valores de la comunidad, acarreando mayor fragmentación y violencia en toda la sociedad. Esta situación, además, se encuentra agravada por la connivencia policial y el narcotráfico, complejizando aún más el diseño de una estrategia integral tendiente a revertir el conflicto. ${ }^{17}$

En los barrios estudiados esta problemática adquiere diferentes niveles de profundidad que permiten delinear cuatro estadios (Cuadro 2): 1. consumo de drogas; 2. consumo y venta de drogas; 3. consumo de drogas y narcomenudeo; y 4. consumo de drogas, narcomenudeo y cocinas de paco o pasta base.

En cada uno de estos estadios aumenta de forma exponencial el compromiso físico, la alienación subjetiva, violencia y consolidación de relaciones de dependencia. Teniendo en cuenta estos niveles, el barrio Costanera es el más comprometido en tanto se ubica en el estadio 4; en este proliferan los suicidios que obedecen al colapso emocional de los sujetos ya sea por la incapacidad de sostener sus crisis o bien por las amenazas constantes de quienes les venden la droga. Al respecto uno de los técnicos barriales comentaba: "[...] este fin de semana se mató otro chico, y un programa del Ministerio fue a la casa a regalarles colchones. La familia los sacó cagando, cómo se les ocurre aparecer asî” (E., Técnico del dispositivo de salud en adicciones, barrio Costanera, 2018).

Este territorio casi liberado solo arroja lecturas negativas en relación con la respuesta estatal sobre esta problemática, frágilmente con-

$17 \mathrm{Al}$ respecto se puede consultar: https://www.eldiario24.com/nota/tucuman/406517/trabajadores-secretaria-adicciones-se-molestaron-declaraciones-yedlin.html; https://www.documentotv.com/ nota/sociedad/32/duro-informe-revelafenomeno-consumo-paco-tucuman.html; http:/ / pcr.org.ar/nota/la-lucha-contra-ladroga-en-tucuman/ 
trarrestada mediante procesos de organización impulsados por grupos en situación de vulnerabilidad extrema. En este sentido, la participación constituye un concepto transversal a las lógicas y prácticas capaces de revertir este orden dominante. Su explicitación, concepción e instrumentación dependen de una adecuada lectura de la multiplicidad de factores que involucra, tales como la manera de ver el mundo y las relaciones de poder en el ámbito que se trate.

En los barrios cuya dinámica participativa gira alrededor de los jóvenes en recuperación de adicciones, es la lucha por espacios públicos destinados a la grupalidad y a la comunidad lo que les permite compartir una tarea factible de ser sostenida junto con el resto del barrio. En los casos de mayor vulnerabilidad, el proyecto se plantea ya no para sí mismos sino para sus hijos, considerando sus propias vidas como ya perdidas.

\begin{tabular}{|c|c|c|c|c|}
\hline \multicolumn{5}{|c|}{ BARRIOS POPULARES } \\
\hline Costanera & Los Vásquez & Santa Inés & 11 de Enero & Diagonal Norte \\
\hline $\begin{array}{l}\text { Consumo de drogas (princi- } \\
\text { palmente paco), narcomenu- } \\
\text { deo y cocinas de paco. Suici- } \\
\text { dios inducidos }\end{array}$ & $\begin{array}{l}\text { Consumo de drogas (princi- } \\
\text { palmente paco), narcome- } \\
\text { nudeo }\end{array}$ & $\begin{array}{l}\text { Consumo de } \\
\text { drogas }\end{array}$ & $\begin{array}{l}\text { Consumo y venta } \\
\text { de drogas (en me- } \\
\text { nor medida) }\end{array}$ & $\begin{array}{l}\text { Consumo de } \\
\text { drogas }\end{array}$ \\
\hline \multicolumn{5}{|c|}{ Desnutrición } \\
\hline \multicolumn{2}{|c|}{ Violencia desencadenada por el narcomenudeo } & \multicolumn{3}{|c|}{ Inseguridad } \\
\hline \multicolumn{5}{|c|}{ Contaminación, anegamiento y precariedad urbana y habitacional } \\
\hline $\begin{array}{l}\text { Ausencia de cualificación de } \\
\text { espacio público }\end{array}$ & $\begin{array}{l}\text { Ausencia de calificación y } \\
\text { cualificación de suelo desti- } \\
\text { nado a espacio público y ser- } \\
\text { vicios }\end{array}$ & $\begin{array}{l}\text { Ausencia de } \\
\text { cualificación } \\
\text { de espacio } \\
\text { público }\end{array}$ & \multicolumn{2}{|c|}{$\begin{array}{l}\text { Ausencia de espacio público y cuali- } \\
\text { ficación del existente }\end{array}$} \\
\hline \multicolumn{2}{|c|}{ Equipamiento comunitario deficitario } & \multicolumn{3}{|c|}{ Ausencia de equipamiento comunitario } \\
\hline
\end{tabular}

\section{Los procesos de mejora participativa del hábitat}

Los casos estudiados transitaron por procesos de participación diferentes, no obstante, comparten ciertas características relacionadas con la temporalidad, el establecimiento de vínculos y los ámbitos de la práctica (Boldrini, 2012) (Cuadro 3). En cuanto a la temporalidad, los antecedentes de cada experiencia junto con las condiciones sociales propias de cada barrio permiten explicar la madurez organizativa alcanzada. Como es de esperarse, los procesos en los que la organización tiene una historia más larga y sostenida alcanzan proyectos más sólidos y factibles de ser concretados. Esto sin embargo no se comprueba en Costanera, donde el deterioro social es tan elevado que obtura el sostenimiento de las actividades, generando interrupciones y dificultades para alcanzar mayor madurez organizativa.

Los vínculos habilitantes para el desarrollo de un proceso participativo donde prima la integración sobre la fragmentación social fueron viables en todos los casos. El deterioro social propio del barrio Costanera dificulta, pero no impide, este tipo de construcción. En todo caso merece la puesta en práctica de más recursos disponibles. En Los Vásquez, donde también predomina el consumo de drogas, la superación de esta problemática parte de propuestas que enlazan dialécticamente instancias individuales, grupales y colectivas. Incluso se han conver-
Cuadro 2. Problemáticas vinculadas con la droga. Fuente: Base de datos Programa de Mejora del Hábitat Participativo. 

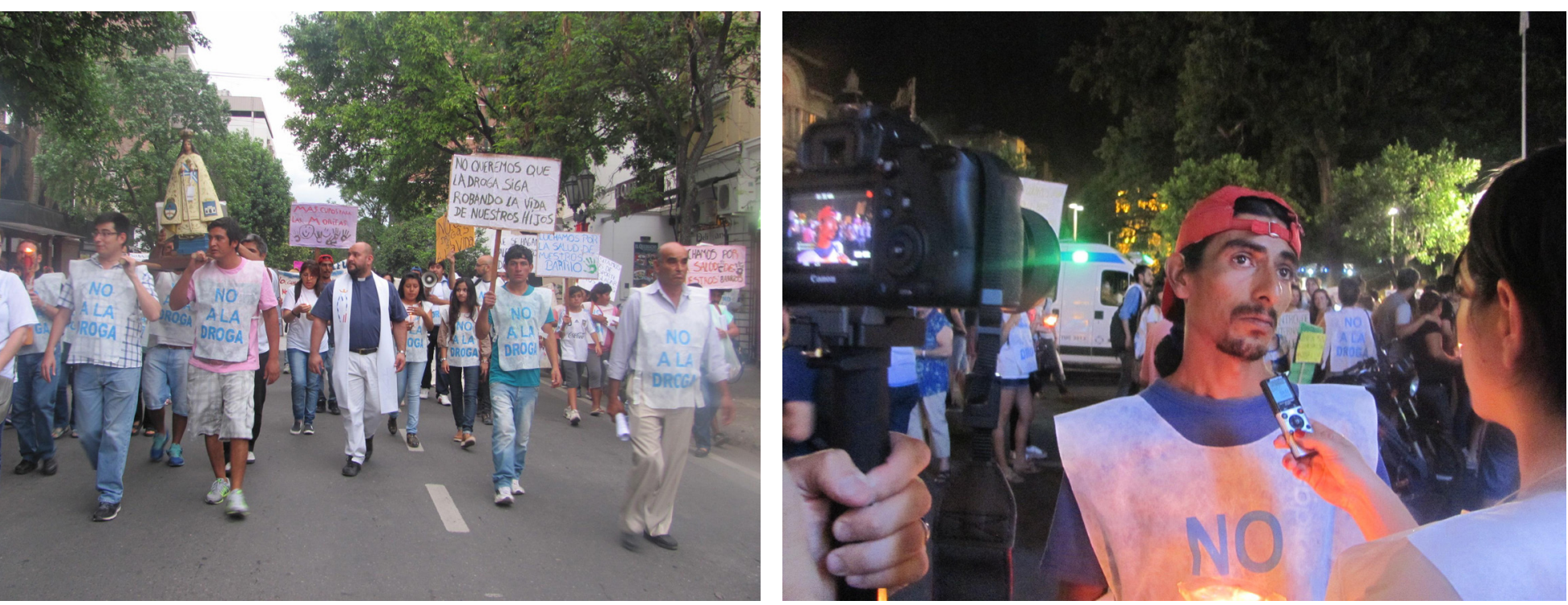

Figura 2. Protesta organizada por la Hermandad de los Barrios para reclamar la inacción del Estado ante el flagelo de las drogas en los barrios vulnerables. Fuente: Base de datos de Boldrini. tido en grupos capaces de promover organizaciones de mayor alcance como la Hermandad de los Barrios que enfrenta el problema del consumo de manera coordinada y asume instancias de repolitización necesarias para revertir desde abajo y estructuralmente los problemas que se presentan (Figura 2).

Los ámbitos en los que se desarrollaron las experiencias analizadas giraron en torno a una dinámica asamblearia, entendida como espacio capaz de contener el surgimiento de necesidades individuales y compartidas, así como modos colectivos de resolución de las mismas. La asamblea estimula la participación, formulación y socialización de problemas y las acciones posibles a emprender. Esta modalidad tiene carácter deliberativo en los casos donde la tarea es acompañada por la organización social La Poderosa (Diagonal Norte y 11 de Enero) y la Asociación Civil Solidaria de Alderetes (Santa Inés); mientras que en los barrios Costanera y Los Vásquez asume un carácter terapéutico, con instancias de enlace con el resto de la comunidad, considerando que el nivel de consumo la afecta directa o indirectamente casi en su totalidad. Son estas instancias las que permiten establecer talleres de producción colectiva de propuestas y proyectos, la organización de la gestión y la protesta social llegado el caso.

Finalmente, el rol de los técnicos fue significativo en todos los casos. Fundamentalmente en aquellos donde se requiere una intervención con carácter terapéutico, el técnico asume un papel central a partir del cual se organiza el resto de las demandas. No obstante, la comunidad protagoniza la formulación de necesidades y mecanismos para obtener soluciones. Los equipos de los dispositivos de salud, liderados por psicólogos especializados en problemas de adicciones, marcan el ritmo de las actividades y los tiempos, en experiencias caracterizadas por integrantes en consumo y acompañados por sus familias. El hábitat se plantea como necesidad y salida inmediata para la resolución de problemas que permitan alcanzar mejores condiciones de salud, garantizado por la perspectiva multicausal desde la cual trabajan los dispositivos. 


\begin{tabular}{|c|c|c|c|c|c|}
\hline \multirow{2}{*}{ VARIABLES } & \multicolumn{5}{|c|}{ BARRIOS POPULARES } \\
\hline & Costanera & Los Vásquez & Santa Inés & 11 de Enero & Diagonal Norte \\
\hline \multirow[t]{3}{*}{ Temporalidad } & $\begin{array}{l}\text { Organización } \\
\text { grupal frágil. Con } \\
\text { experiencias pre- } \\
\text { vias. El abordaje } \\
\text { se realiza por sec- } \\
\text { tores que se arti- } \\
\text { culan en función } \\
\text { de las demandas }\end{array}$ & $\begin{array}{l}\text { Organización } \\
\text { grupal sólida. El } \\
\text { abordaje se rea- } \\
\text { liza mediante di- } \\
\text { versas experien- } \\
\text { cias que crecen } \\
\text { en complejidad }\end{array}$ & $\begin{array}{l}\text { Grupo con an- } \\
\text { tecedentes de } \\
\text { organización au- } \\
\text { togestiva para el } \\
\text { completamien- } \\
\text { to de tareas. Di- } \\
\text { námica madura } \\
\text { para la ejecución } \\
\text { de actividades }\end{array}$ & $\begin{array}{l}\text { Organización ba- } \\
\text { rrial incipiente. } \\
\text { Sin experiencias } \\
\text { de trabajo comu- } \\
\text { nitarias previas }\end{array}$ & $\begin{array}{l}\text { Organización } \\
\text { barrial madura. } \\
\text { Grupo de Vetera- } \\
\text { nos con } 40 \text { años } \\
\text { de antigüedad. } \\
\text { Diversas expe- } \\
\text { riencias que cre- } \\
\text { cen en comple- } \\
\text { jidad }\end{array}$ \\
\hline & $\begin{array}{l}\text { El dispositivo de } \\
\text { salud coordina } \\
\text { actividades desde } \\
\text { el año } 2012\end{array}$ & $\begin{array}{l}\text { El dispositivo de } \\
\text { salud coordina } \\
\text { actividades desde } \\
\text { el año } 2015\end{array}$ & $\begin{array}{l}\text { El dispositivo de } \\
\text { salud coordina } \\
\text { actividades desde } \\
\text { el año } 2016\end{array}$ & $\begin{array}{l}\text { La Poderosa } \\
\text { coordina activida- } \\
\text { des desde el año } \\
2017\end{array}$ & $\begin{array}{l}\text { La Poderosa } \\
\text { coordina activida- } \\
\text { des desde el año } \\
2007\end{array}$ \\
\hline & $\begin{array}{l}\text { El Equipo MHa- } \\
\text { Pa trabaja desde } \\
\text { el año } 2017\end{array}$ & $\begin{array}{l}\text { El Equipo MHa- } \\
\text { Pa trabaja desde } \\
\text { el año } 2015\end{array}$ & \multicolumn{2}{|c|}{$\begin{array}{l}\text { El Equipo MHaPa trabaja desde el } \\
\text { año } 2017\end{array}$} & $\begin{array}{l}\text { El Equipo MHa- } \\
\text { Pa trabaja desde } \\
\text { el año } 2016\end{array}$ \\
\hline $\begin{array}{l}\text { Construcción } \\
\text { de vínculos }\end{array}$ & $\begin{array}{l}\text { Relaciones in- } \\
\text { constantes entre } \\
\text { vecinos. Víncu- } \\
\text { los sólidos con } \\
\text { técnicos de los } \\
\text { Dispositivos e in- } \\
\text { cipientes con los } \\
\text { del MHaPa. Rela- } \\
\text { ciones desiguales } \\
\text { con PROMEBA }\end{array}$ & $\begin{array}{l}\text { Madurez vincular } \\
\text { entre los jóvenes, } \\
\text { con los técnicos } \\
\text { de los Dispositi- } \\
\text { vos y con los del } \\
\text { MHaPa }\end{array}$ & $\begin{array}{l}\text { Relaciones in- } \\
\text { constantes entre } \\
\text { vecinos. Vínculos } \\
\text { establecidos entre } \\
\text { Asociación Civil } \\
\text { Solidaria de Alde- } \\
\text { retes, técnicos de } \\
\text { los Dispositivos y } \\
\text { del MHaPa }\end{array}$ & $\begin{array}{l}\text { Vínculos inci- } \\
\text { pientes entre ve- } \\
\text { cinos, técnicos de } \\
\text { La Poderosa y del } \\
\text { MHaPa }\end{array}$ & $\begin{array}{l}\text { Madurez vincular } \\
\text { entre los vecinos, } \\
\text { con técnicos de } \\
\text { La Poderosa y del } \\
\text { MHaPa }\end{array}$ \\
\hline $\begin{array}{l}\text { Ámbitos de } \\
\text { trabajo }\end{array}$ & \multicolumn{2}{|c|}{$\begin{array}{l}\text { Trabajo terapéutico en torno al cual } \\
\text { se desarrollan las tareas y se convoca } \\
\text { al resto del barrio. Dinámica asam- } \\
\text { blearia con talleres, relevamiento y } \\
\text { trabajo de gestión complementaria. } \\
\text { Protesta social: "Hermandad de los } \\
\text { Barrios" }\end{array}$} & \multicolumn{2}{|c|}{$\begin{array}{l}\text { Dinámica asamblearia con talleres, re- } \\
\text { levamiento y trabajo de gestión com- } \\
\text { plementaria. }\end{array}$} & $\begin{array}{l}\text { Dinámica asam- } \\
\text { blearia con talle- } \\
\text { res, relevamiento } \\
\text { y trabajo de ges- } \\
\text { tión complemen- } \\
\text { taria. Protesta so- } \\
\text { cial. }\end{array}$ \\
\hline
\end{tabular}

\section{Lineamientos estratégicos para las lógicas y prácticas participativas implementadas en barrios populares}

Teniendo en cuenta que este artículo propone que aquellas estrategias identificadas como más apropiadas para trabajar en la mejora integral del hábitat se conviertan en un insumo teórico-instrumental que sustente las lógicas y prácticas participativas implementadas en barrios populares, se plantean los siguientes lineamientos estratégicos:

a) Construir equipos interdisciplinarios, intersectoriales e interinstitucionales cuya sinergia permite establecer pautas y mecanismo de trabajo apropiadas al nuevo contexto social.

b) Formar a los técnicos que trabajan en el territorio provenientes de distintas disciplinas (psicología, psicología social, trabajo social, arquitectura, entre otras) con una perspectiva que ponga especial atención en las problemáticas actuales y en las distintas instancias de trabajo individual, familiar, grupal y colectivo. Asimismo, es preciso estar atentos a la identificación de nuevas pro-
Cuadro 3. Instancias del proceso participativo. Fuente: Base de datos del Programa de Mejora del Hábitat Participativo. 
blemáticas emergentes que requieran la incorporación de otras disciplinas.

c) Construir vínculos con los sujetos en situación de adicción y abandono, proponiendo la conformación de grupos por afinidad (jóvenes, mujeres, madres, entre otros). Esta modalidad permite, a partir de la problemática compartida en adicciones, contribuir al inicio de nuevas relaciones intra-barriales.

d) Promover actividades y proyectos que contribuyan a la conformación y consolidación de los grupos constituidos, cuidando que mantengan cierto grado de proyección comunitaria. Este aspecto contribuye a la reinserción a mayor escala de los enfermos adictos, frecuentemente subestimados y estigmatizados, como sujetos con capacidad transformadora.

e) Sostener procesos a lo largo del tiempo, trascendiendo los altibajos propios de los procesos participativos de manera tal de acompañar la resolución de demandas y surgimiento de nuevas necesidades que motoricen el trabajo colectivo.

f) Generar ámbitos de trabajo donde prevalezca el establecimiento de vínculos de confianza e inclusión, en los que se respeten los modos de pensar, sentir y actuar en sus diferentes lenguajes y manifestaciones.

g) Plantear la mejora integral del hábitat como necesidad y salida inmediata para la resolución de problemas que permitan alcanzar mejores condiciones de salud, respetando la perspectiva multicausal de trabajo en el territorio (en este caso propuesta por los técnicos de los dispositivos de salud). Este abordaje permite al equipo técnico-académico territorial acceder a los aspectos centrales de cada barrio, superando la estrategia de investigación cualitativa, conociendo la vida cotidiana de sus habitantes y trabajando activamente en la co-construcción de cada uno de estos procesos de mejora.

\section{Reflexiones finales}

Teniendo en cuenta las condiciones actuales del contexto social, se hace necesario diferenciar estrategias de intervención apropiadas según las problemáticas inherentes a cada barrio pero que, a su vez, tengan en cuenta las circunstancias vinculadas al contexto urbano más amplio en que están insertos. A los aspectos tradicionales que se tienen en cuenta al estudiar diversas situaciones en los barrios populares (variables poblacionales, laborales, socio-económicas y habitacionales, entre otras), se agrega en la actualidad la problemática de las adicciones, que representa una preocupación y, por ende, una iniciativa y motor central de trabajo. Este fenómeno representa un salto cualitativo en el empeoramiento de las condiciones de vida de los grupos poblaciones más empobrecidos y de la comunidad en general.

Las políticas públicas actuales, lejos de intervenir para revertir las condiciones estructurales y actuales que afrontan los sectores populares, tienden a perpetuarlas, obstaculizando los procesos participativos y generando condiciones cada vez más complejas y difíciles de superar ante el creciente nivel de alienación de los sujetos, el desgaste de los grupos y el deterioro de las redes sociales. Las experiencias analizadas dan cuenta de que es posible trabajar en el territorio con otras lógicas, dinámicas y estrategias de intervención. Estas deben poner en un lugar 
protagónico a las comunidades y a la construcción de los vínculos habilitantes para el desarrollo de procesos participativos donde prima la integración sobre la segregación social.

Se hace evidente que esta tarea merece una rigurosa formación de los técnicos que trabajan en el territorio - particularmente en el trato con sujetos en consumo- por lo que la presencia de los técnicos (provenientes de distintas disciplinas) debe estar garantizada por parte del Estado y constituirse como política pública a fin de que su intervención sea sistemática y continuada a través del tiempo. En este sentido se destaca el Proyecto de Ley de Acompañamiento Técnico, Profesional y Público Gratuito impulsado desde la Red Habitar Argentina, ${ }^{18}$ que condensa el sentir y hacer de profesionales preocupados y ocupados en la mejora del hábitat junto a los sectores populares. Esta propuesta institucionaliza la presencia de los profesionales en los barrios como trabajadores del Estado, cuyas prácticas se desarrollan en el campo concreto, y contribuye a la configuración de nuevos perfiles y roles profesionales e intelectuales, habitualmente apartados de las dinámicas populares y escindidos de la producción científica efectivamente útil. La práctica se produce en un encuentro dialéctico con la producción de teoría, tanto desde el planteo de temas de investigación relevantes como de insumos de investigación que permiten mayor profundidad de análisis.

La preocupación recurrente y sostenida por los habitantes de los barrios populares se vincula tanto con la proliferación de problemáticas relacionadas con la droga como con la urgente mejora del espacio púbico (como espacio de recreación), y pone en evidencia el interés y valoración del componente colectivo de la vida cotidiana. En este sentido los procesos estudiados constituyen dinámicas de des-reterritorialización en -la mayoría de las veces- una violenta puja entre la cooptación del espacio público por parte de agentes delictivos y la resistencia de la comunidad organizada que busca generar y recuperar espacios de uso social. De este modo la cualificación del espacio público como estrategia de trabajo en el territorio (muy utilizado en planes de mejora a diferentes escalas) continúa ocupando un lugar central. Da cuenta - en un contexto de patologías complejas como las adicciones- de aquellos aspectos de salud que detenta la comunidad, entendida como capacidad de adaptarse activamente a la realidad, transformándola y transformándose a sí mismos (Pichon Rivière, 1982).
18 Ver en línea: http://www.habitarargentina.org.ar/proyectos-de-ley. 


\section{Referencias bibliográficas}

Althabe, Gerard (1999). Lo microsocial y la investigación antropológica de campo. En Althabe, Gerard y Schuster, Félix (eds.), Antropología del presente (pp. 11-21). Buenos Aires: Editorial Edicial.

Auyero, Javier (2002). La protesta. Retratos de la beligerancia popular en la argentina democrática. Buenos Aires: Libros del Rojas.

Boldrini, Paula (2011). Vinculo entre comunidad y arquitectos en una experiencia de producción de hábitat en Tucumán (Tesis de maestría). Facultad de Psicología (UNT), Tucumán.

Boldrini, Paula (2012). Producción participativa del hábitat popular en el área metropolitana de Tucumán (Tesis doctoral). Facultad de Filosofía y Letras (UNT), Tucumán.

Boldrini, Paula (2018). Informalidad en el Gran San Miguel de Tucumán. En Cravino, Cristina (ed.), La ciudad (re)negada. Aproximaciones al estudio de asentamientos populares en nueve ciudades argentinas (pp. 127-149). Buenos Aires: Universidad General Sarmiento.

Boldrini, Paula; Del Castillo, Alejandra; y Malizia, Matilde (2014). Condiciones de vida y fragmentación socio-espacial en el aglomerado Gran San Miguel De Tucumán (noroeste Argentino). Estudios Socioterritoriales, 15, 15-43.

Boldrini, Paula; Malizia, Matilde; y Paolasso, Pablo (2018). El análisis intra-urbano: fragmentación y crecimiento en islas. En Malizia, Matilde; Boldrini, Paula y Paolasso, Pablo; (eds.), Hacia otra ciudad posible. Transformaciones urbanas recientes en el aglomerado Gran San Miguel de Tucumán (pp. 47-86). Buenos Aires: Café de las ciudades.

Bolsi, Alfredo; y Paolasso, Pablo (2009). Geografía de la pobreza en el Norte Grande Argentino. San Miguel de Tucumán: PNDU / UNT.

Castells, Manuel (1985). Estado, cultura y sociedad: las nuevas tendencias históricas. Madrid: Ministerio de Cultura. Cultura y Sociedad.

Castells, Manuel (1968). Y a-t-il une sociologie urbaine? Sociologie du Travail, 1, 72-90.

Ciccolella, Pablo (2010). La ciudad mestiza: metrópolis latinoamericanas atrapadas entre la globalización y la inclusión social. Tamoios, 6(2), 4-16. DOI: 10.12957/tamoios.2010.1414.

Coulomb, René (1992). Pobreza urbana, autogestión y política. México: Editorial del Centro de Vivienda y Estudios Urbanos.

Cravino, Cristina; y Varela, Omar (2008). Los mil barrios (in)formales en el Área Metropolitana de Buenos Aires. Los Polvorines: Universidad de General Sarmiento.
Cuozzo, Rosa Lina; Boldrini, Paula; y Malizia, Matilde (2018). El crecimiento metropolitano (1990-2015). En Malizia, Matilde; Boldrini, Paula; y Paolasso, Pablo (eds.), Hacia otra ciudad posible. Transformaciones urbanas recientes en el Aglomerado Gran San Miguel de Tucumán (pp. 87-112). Buenos Aires: Café de las ciudades.

Décima, Débora (2019). Discursividad mediática sobre el acceso y producción del hábitat popular en el barrio Costanera. Ponencia presentada en el I Encuentro de Asentamientos informales. Facultad de Arquitectura, Urbanismo y Diseño, Universidad Nacional de Córdoba. Argentina.

Duhau, Emilio (1998). Hábitat popular y política urbana. México: Miguel Ángel Porrúa y UAM-Azcapotzalco.

Del Castillo, Alejandra (2012). Pobreza y 'cartoneo' en un barrio periférico del Gran San Miguel de Tucumán. Cuadernos de Humanidades, 41, 249-271.

Enet, Mariana (2012). Diseño participativo: Estrategia efectiva para el mejoramiento ambiental y economía social en viviendas de baja renta. Cuadernos de Vivienda y Urbanismo, 5(10), 198-233.

Fernandes, Edésio y Smolka, Martim (2004). Land regularization and upgrading programs revisited. Land Lines, 16. Recuperado el 2 de febrero de 2020 de https://www.lincolninst.edu/es/publications / articles/regularizacion-la-tierra-programas-mejoramiento.

Gómez López, Claudia; Cuozzo Rosa Lina; y Boldrini, Paula (2012). Expansión urbana y desigualdades socio- territoriales en el Área Metropolitana de Tucumán. Argentina. Ponencia presentada en el Coloquio de Transformaciones Territoriales AUGM. Facultad de Arquitectura y Urbanismo, Universidad Nacional de Tucumán. Argentina.

Gómez López, Claudia; Cuozzo Rosa Lina; y Boldrini, Paula (2015). Impactos de las Políticas de Vivienda en el Área Metropolitana de Tucumán, Argentina. 2003-2013. Cuaderno Urbano, 19(19), 153-178.

Haesbaert, Rogério (2011). Del mito de la desterritorialización a la multiterritorialidad. Cultura y representaciones sociales, 8(15), 9-42. Recuperado el 29/03/2020 de: http:/ /www.culturayrs.unam.mx/ index.php/CRS/article/view/401.

Guber, Rosana (2009). El salvaje metropolitano. Buenos Aires: Legasa.

Harvey, David (2013). Ciudades rebeldes. Del derecho a la ciudad a la revolución urbana. Buenos Aires: Akal, Pensamiento crítico.

Janoschka, Michael (2011). Geografías urbanas en la era del neoliberalismo. Una conceptualización de la resistencia local a través de la participación y la 
ciudadanía urbana. Investigaciones Geográficas, 76, 118-132. DOI: 10.14350/rig.29879.

Llovera, María Soledad, y Scialla, Marina (2017). Políticas de drogas en Argentina (2003-2015). Reflexiones en torno a la puja entre lo heredado y lo reformulado. Temas y Debates, 34, 77-99. DOI: 10.35305/ tyd.v0i34.370.

Malizia, Matilde (2011). Countries y barrios privados en el Gran San Miguel de Tucumán. Efectos y contrastes sociales (Tesis doctoral). Facultad de Filosofía y Letras (UNT), Tucumán.

Malizia, Matilde; y Boldrini, Paula (2012). Las lógicas de ocupación del espacio urbano. Un estudio de realidades contrapuestas. El caso de las urbanizaciones cerradas y villas miseria en yerba buena, Gran San Miguel de Tucumán. Cuadernos de Humanidades, 41, 197-219.

Mendizábal, Nora (2019). El otro río: las investigaciones en ciencias sociales realizadas con métodos mixtos. En Vasilachis de Gialdino, Irene (eds.), Estrategias de investigación cualitativa. Volumen II (pp.241-286). Buenos Aires: Gedisa Editorial.

Neiman, Guillermo y Quaranta, Germán (2006). Los estudios de caso en la investigación sociológica. En Vasilachis de Gialdino, Irene (eds.), Estrategias de investigación cualitativa. Volumen I (pp. 213-238). Buenos Aires: Gedisa Editorial.

Neder, Liliana; Caria, Mario; y Busnelli, José (2008). Influencia de factores naturales y antropogénicos en el diseño de drenaje fluvial en áreas urbanas. Tucumán: Facultad de Ciencias Naturales e Instituto Miguel Lillo, Universidad Nacional de Tucumán.

Osatinsky, Ariel; y Paolasso, Pablo (2012). La industria en la provincia de Tucumán: de la expansión azucarera a la desindustrialización. En Bergesio, Liliana; y Golovanevsky, Laura (eds.), Industria y Sociedad. El sector manufacturero en Jujuy y Argentina (pp. 67-100). Jujuy: EdiUnju, Colección Economía, trabajo y producción.

Paolasso, Pablo; Del Castillo, Alejandra; Malizia, Matilde; y Boldrini, Paula (2013). Vulnerabilidad e Informalidad urbana en el Gran San Miguel de Tucumán (NW Argentina). En Sandia Rondón, Luis Alfonso (ed.), Grandes ciudades latinoamericanas: informalidad y pobreza, viejas y nuevas formas y sus impactos en la gobernabilidad. Venezuela: Universidad de Los Andes, Universidad Bolivariana de Venezuela, Instituto de Geografía Tropical, Universidad Nacional de Tucumán, Universidad Federal de Pernambuco y la Universidad de Marburg.
Paolasso, Pablo; Malizia, Matilde; y Longhi, Fernando (2011). Vulnerabilidad y segregación socio-espacial en el Gran San Miguel de Tucumán (Argentina). En Vergara Durán, Adrián (ed.), Vulnerabilidad en grandes ciudades de América Latina (pp. 50-71). Barranquilla: Ediciones Uninorte.

Pascale, Antonio; Hynes, Marya; Cumsille, Francisco y Bares, Cristina (2014). Consumo de pasta base de cocaína en América del Sur. Revisión de los aspectos epidemiológicos y médico-toxicológicos. Montevideo: OEA-CICAD.

Pelli, Victor (2007). Habitar, Participar, Pertenecer. Acceder a la vivienda-incluirse en la sociedad. Buenos Aires: Nobuko.

Pichon Rivière, Enrique (1982). El proceso grupal, del psicoanálisis a la psicología social (1). Buenos Aires: Ediciones Nueva Visión.

Pichon-Rivière, Enrique (1999). El proceso grupal. Buenos Aires: Ediciones Nueva Visión.

Pradilla Emilio (2010). Mundialización neoliberal, cambios urbanos y políticas estatales en América Latina. Cadernos Metrópole, 2(24), 507-533. DOI: 10.15332/rev.m.v6i2.1021.

Pradilla, Emilio (1984). Contribución a la crítica de la teoría urbana. Del espacio a la crisis urbana. México: Universidad Autónoma Metropolitana, Unidad Xochimilco.

Purcell, Mario (2007). City-Regions, neoliberal globalization and democracy: a research agenda. International Journal of Urban and Regional Research, 31, 197206. DOI: 10.1111/j.1468-2427.2007.00714.x.

Svampa Maristella (2008). Notas provisorias sobre la sociología, el saber académico y el compromiso intelectual. En Althabe, Gerard (ed.), Entre dos mundos. Reflexividad y compromiso (pp. 86-103). Buenos Aires: Prometeo.

Vasilachis de Gialdino, Irene (2006). Estrategias de Investigación cualitativa. Volumen I. Buenos Aires: Gedisa Editorial.

Velázquez, Guillermo; Mikkelsen, Claudia; Linares, Santiago y Celemín, Juan Pablo (2014). Calidad de vida en la Argentina: ranking del bienestar por departamentos (2010). Tandil: Universidad Nacional del Centro de la Provincia de Buenos Aires.

Yuni, José Alberto y Urbano, Claudio Ariel (2003) Recursos metodológicos para la preparación de proyectos de investigación. Volumen II. Córdoba, Argentina: Editorial Brujas. 
Boldrini, Paula; y Malizia, Matilde (2020). Mejora participativa del hábitat en contextos de desigualdad en ciudades intermedias. Hábitat y Sociedad, 13, 209-228.

<http:/ /dx.doi.org/10.12795/HabitatySociedad.2020.i13.12> 